\title{
Interferon-gamma-expressing Adenovirus Vaccine ASN-002
}

National Cancer Institute

\section{Source}

National Cancer Institute. Interferon-gamma-expressing Adenovirus Vaccine ASN-002.

NCI Thesaurus. Code C66972.

A replication-defective adenoviral serotype 5 vector encoding a recombinant form of the human cytokine interferon-gamma (IFN-g), with potential antineoplastic and immunoregulatory activities. Upon intratumoral administration, the sustained expression of IFN-g by IFN-g-expressing adenovirus vaccine ASN-002 promotes a T-helper type 1 (Th1) immune response and inhibits the Th2-mediated cytokine production observed in many cutaneous lymphomas. IFN-g also mediates interleukin-12 (IL-12) production by antigen-presenting cells (APCs); activates macrophages, cytotoxic T-cells, and natural killer (NK) cells; upregulates major histocompatibility complex (MHC) molecules; and stimulates antibody-dependent cellular cytotoxicity (ADCC). Altogether, these IFN-gmediated effects may result in both an inhibition of tumor cell proliferation and tumor cell death. Compared to IFN-g injections, the prolonged local production of IFN-g at the tumor site allows for higher efficacy and a reduction of systemic toxicity. 